\title{
BMJ Open Text messages for primary prevention of cardiovascular disease: the TextMe2 randomised controlled trial protocol
}

\author{
Harry Klimis (D) , ${ }^{1,2}$ Aravinda Thiagalingam, ${ }^{1,2}$ Clara K Chow ${ }^{1,2}$
}

To cite: Klimis $\mathrm{H}$, Thiagalingam A, Chow CK. Text messages for primary prevention of cardiovascular disease: the TextMe2 randomised controlled trial protocol. BMJ Open 2020;10:e036767. doi:10.1136/ bmjopen-2020-036767

- Prepublication history and additional material for this paper are available online. To view these files, please visit the journal online (http://dx.doi. org/10.1136/bmjopen-2020036767).

Received 02 January 2020 Revised 27 March 2020 Accepted 07 April 2020

\section{Check for updates}

\section{(c) Author(s) (or their} employer(s)) 2020. Re-use permitted under CC BY-NC. No commercial re-use. See rights and permissions. Published by BMJ.

${ }^{1}$ Westmead Applied Research Centre, Faculty of Medicine and Health, The University of Sydney, Sydney, New South Wales, Australia

${ }^{2}$ Department of Cardiology, Westmead Hospital, Westmead, New South Wales, Australia

Correspondence to

Dr Harry Klimis;

harry.klimis@sydney.edu.au

\begin{abstract}
Introduction Mobile health may be an effective means of delivering customised individually directed health promotion interventions for cardiovascular disease (CVD) primary prevention. The aim of this study is to evaluate the effectiveness of a lifestyle-focused text messaging programme for primary CVD prevention.

Methods and analysis Single-blind randomised controlled trial with 6 months' follow-up in 246 patients with moderate-high absolute cardiovascular risk and without coronary heart disease recruited from a rapid access cardiology clinic. Participants will be randomised to receive either usual care or TextMe2 (text messagebased prevention programme). The TextMe2 programme provides support, motivation and education on five topics: diet, physical activity, smoking, general cardiovascular health and medication adherence, and is delivered in four text messages per week over 6 months. The primary outcome is change in the proportion of patients who have three or more of five key modifiable risk factors that are uncontrolled (low-density lipoprotein $>2.0 \mathrm{mmol} / \mathrm{L}$, systolic blood pressure $>140 \mathrm{~mm} \mathrm{Hg}$, body mass index $>24.9$ $\mathrm{kg} / \mathrm{m}^{2}$, physical activity (less than the equivalent of 150 min of moderate intensity each week), current smoker). Secondary outcomes are changes in single biomedical risk factors, behavioural risk factors, quality of life, depression/ anxiety scores, medication adherence, cardiovascular health literacy and hospital readmissions/representations. Analysis will be according to the intention-to-treat principle and full statistical analysis plan developed prior to data lock.
\end{abstract}

Ethics and dissemination This study has been approved by the Western Sydney Local Health District Human Research Ethics Committee at Westmead (AU/ RED/HREC/17/WMEAD/186). Results will be presented at scientific meetings and published in peer-reviewed publications.

Trial registration number ACTRN12618001153202.

\section{INTRODUCTION}

Cardiovascular disease (CVD) is the largest cause of global premature death and disability. ${ }^{2}$ Abnormal lipids, high blood pressure, smoking, excess alcohol consumption, physical inactivity, diabetes, abdominal obesity, psychosocial factors and fruit/vegetable consumption account for the majority of population attributable risk of myocardial

\section{Strengths and limitations of this study}

- This study will be unique in being a randomised clinical trial evaluation compared with usual care of the effectiveness of an entirely text message-based intervention for managing multiple cardiovascular risk factors in a primary prevention setting.

- Intervention participants will receive the texting intervention and control participants will receive usual care and thus not blinded; however, outcome assessors will be blinded to participant allocation.

- TextMe2 is a single-centre study, offered to participants at moderate-high absolute cardiovascular risk presenting to a large Western Sydney hospital serving a culturally and economically diverse population in Australia and may hence not be generalisable to all settings.

- Outcome measures will be a combination of objective and self-reported risk factor levels, for example, smoking, diet, physical activity and medication adherence. Self-report may not reflect actual behaviour; however, the standardised instruments will be applied to all participants to minimise bias.

infarction worldwide and key is that all of these are modifiable risk factors. ${ }^{3}$

Current primary prevention guidelines emphasise the importance of both lifestyle modification as well as relevant pharmacotherapy for high blood pressure and high low-density lipoprotein-cholesterol (LDL-C) in people at moderate-high absolute cardiovascular risk. ${ }^{4}$ Provision of cardiovascular risk assessment and a CVD risk score in the primary prevention setting can result in small but significant improvements in risk factor control (lipid levels, blood pressure, smoking cessation and multivariable CVD risk) and leads to increased preventive medication prescription (lipid-lowering therapy, blood pressure-lowering therapy and aspirin initiation) in higher risk individuals. ${ }^{6}$ However, changing and maintaining healthy lifestyle changes and medications can be challenging as can be achieving levels of intensive risk factor control. 
Barriers to lifestyle and medication adherence for primary prevention exist at the patient, healthcare provider and healthcare system level and include: lack of motivation, prescribing complex regimens, poor clinician-patient communication and poor access and availability of healthcare. ${ }^{7}$ Thus, high-quality research into novel cost-effective and accessible prevention programmes targeting, and successfully engaging, individuals at moderate-high absolute cardiovascular risk (ie, a 10-year risk of a CVD event $\geq 10 \%$ based on Framingham risk equation) is needed.

The use of mobile health (mHealth) technology is a potential method of delivering prevention programmes and improving engagement with chronic disease prevention strategies. mHealth includes short messaging service (SMS or referred to as text messages), as well as more complex functionalities and mobile applications. Due to its ubiquity, ${ }^{8}$ this technology may reduce access to healthcare and allow convenient community-based health assessment, support the exchange of health information and encourage positive health behaviour. ${ }^{910}$

A systematic review has shown text message-based interventions to be effective in CVD secondary prevention, ${ }^{11}$ and cost-effectiveness has also been demonstrated ${ }^{12}$ in this population. The ubiquity of phones and the potential for language and cultural customisation mean they have great potential to be widely disseminated, bridging geographical, cultural and financial barriers. ${ }^{13}{ }^{14}$ Recent studies exemplify how text message-based intervention programmes can feasibly be implemented even in low and middle-income countries across a range of geographical and cultural settings. ${ }^{13-15}$ Systematic reviews assessing the effects of text messaging on isolated cardiovascular risk factors have shown benefits including smoking cessation, ${ }^{16}$ weight loss, ${ }^{17}$ diabetes ${ }^{18}$ and medication adherence in chronic disease. ${ }^{19}$ Importantly, most studies have focused on single risk factor control and there is evidence that programmes targeting multiple risk factors may be more effective and more cost-effective than treatment decisions based on individual risk factor targets. ${ }^{11} 20$ However, text messaging for multiple risk factor control in the primary prevention setting has not been extensively assessed.

There is some evidence demonstrating the impact of text message-based prevention programmes on multiple risk factor control in secondary prevention. ${ }^{11} 20$ The Tobacco, Exercise and Diet Messages (TEXT ME) study demonstrated in a single-centre randomised controlled trial (RCT) that multiple cardiovascular risk factors could be targeted and impacted by a programme of text messages that addressed secondary prevention of CVD in patients with coronary heart disease. Specifically, it demonstrated that more people in the intervention group achieved four or more out of five risk factor targets (smoking cessation, LDL-C $<77 \mathrm{mg} / \mathrm{dL}$, blood pressure $<140 / 90 \mathrm{~mm} \mathrm{Hg}$, regular physical activity and body mass index (BMI) $<25 \mathrm{~kg} / \mathrm{m}^{2}$ ) compared with control (RR $2.8,95 \%$ CI 1.95 to $4.02, \mathrm{p}<0.001) .{ }^{21}$ Similar findings have also been demonstrated in a recent multicentre RCT from China (the Cardiovascular Health and Text Messaging (CHAT) trial) again targeting a secondary prevention cohort. The CHAT trial ${ }^{14}$ showed that a text message-based prevention programme resulted in more participants achieving three or more guideline-based risk factor targets (RR 1.2, 95\% CI 1.1 to $1.3, \mathrm{p}=0.004$ ), despite no significant changes in individual risk factor control including their primary outcome of blood pressure. This highlights the importance of designing studies with the primary outcome addressing multiple risk factors to achieve cardiovascular risk reduction.

There have been limited (two) RCTs ${ }^{22} 23$ demonstrating the effectiveness of text message-based approaches targeting multiple cardiovascular risk factors in a primary prevention setting, and no RCTs on smartphone-based interventions. ${ }^{11}$ The largest delivered a 12 -month intervention consisting of both text messages and phone calls (frequency varied between once per month and once per week) to 589 Chinese workers. This showed a reduction in blood pressure, BMI, fasting blood sugar level, waist-to-hip ratio and total cholesterol for the intervention group at 12 months compared with baseline. However, there was no difference in total CVD risk score at 12 months compared with baseline.$^{23}$ The second study delivered a 6-month programme to Asian adults living in Canada via either SMS (delivered weekly) or email (delivered every two weeks) decided by participant choiceless than $8 \%$ of participants chose SMS. ${ }^{22}$ There was no significant difference in overall myocardial infarction risk scores between intervention and control groups at 12-month follow-up. These studies were heterogeneous in frequency of delivery and level of interaction, and used mixed modalities (eg, SMS and email). ${ }^{22}{ }^{23}$ Thus, it is difficult to draw conclusions and we need trials showing how patients interact with these interventions in order to optimise future mHealth interventions, and evaluate whether these digital health interventions would be effective in a primary prevention cohort, which may be more difficult to engage. Patients who have been referred to an outpatient cardiology service for assessment of chest pain ${ }^{24} 25$ and calculation of a cardiovascular risk score are more likely to be engaged with primary prevention strategies such as lifestyle changes and medication adherence. ${ }^{6}$

The primary objective of the TextMe2 study is to determine the impact of a programme of text messages on multiple modifiable cardiovascular risk factors, in moderate-high absolute cardiovascular risk individuals who have been referred to outpatient cardiology services for chest pain but without documented coronary artery disease (CAD) (ie, primary prevention cohort). In addition, this study will look at the effect of such a programme on quality of life, health literacy, medication adherence and depression/anxiety scores. We wanted to: (1) examine if mHealth interventions used in a secondary prevention setting can be applied in primary prevention setting with good effect, (2) provide an alternative and scalable primary prevention strategy, (3) identify opportunities 


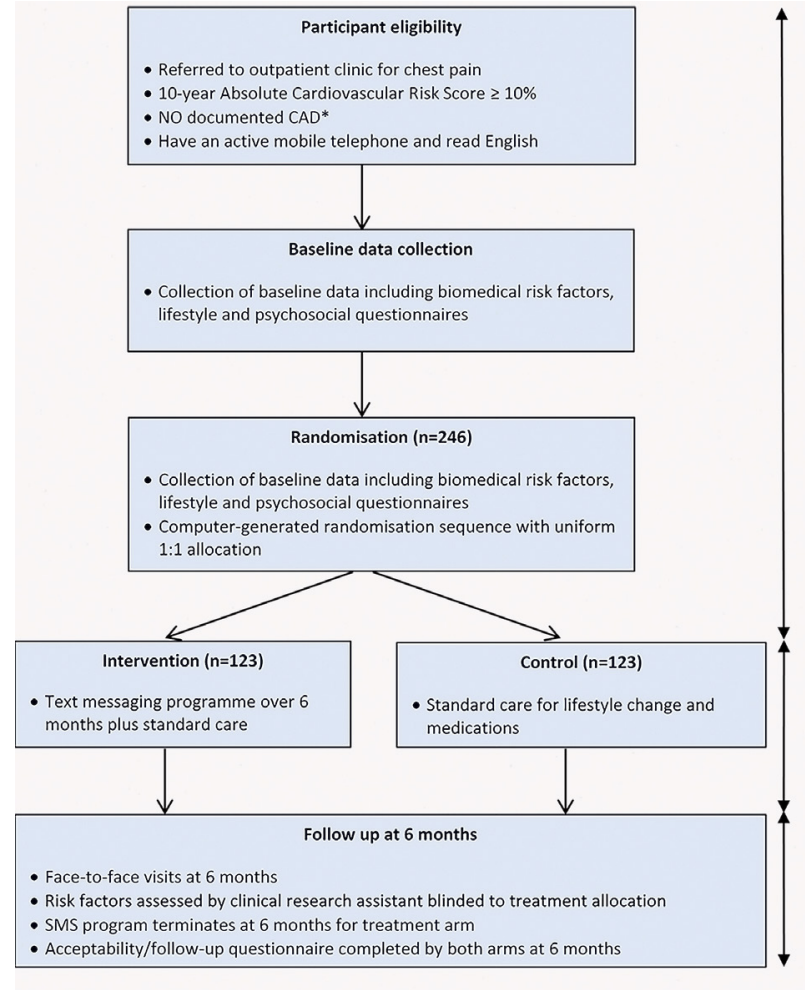

Figure 1 Study design and flow. CAD, coronary artery disease; SMS, short messaging service.

for delivering primary prevention programmes at the clinical interface in order to improve engagement with such programmes, and (4) examine participant rating systems for individual text messages to explore whether messaging can be optimised in the future with such rating systems.

\section{METHODS AND ANALYSIS Design}

TextMe2 is a single-blind RCT with 6 months' follow-up evaluating a text message-based primary prevention programme compared with usual care (in a 1:1 allocation ratio) in people at moderate-high absolute cardiovascular risk from a rapid access cardiology clinic (RACC) on the primary outcome of change in the proportion of patients who have three or more uncontrolled modifiable risk factors (figure 1). The TextMe2 programme will deliver multiple weekly text messages providing support, motivation and education on cardiac prevention.

\section{Study setting}

This single-site study will be conducted at a large Australian tertiary referral centre that serves an ethnically, culturally and socioeconomically diverse population. Participants will be recruited from an RACC that is colocated within the hospital and previously described. ${ }^{24}{ }^{25} \mathrm{In}$ brief, RACCs provide early assessment and management of low to intermediate-risk chest pain including cardiovascular risk assessment.

\section{Study population}

Participants will be identified from patients referred to the RACC for chest pain at a large quaternary hospital in Sydney, Australia. Patients will be eligible if they are adult ( $\geq 18$ years), have a moderate-high cardiovascular risk ( $\geq 10 \%$ 10-year risk) and do not have a history of coronary heart disease. Absolute cardiovascular risk will be estimated using a clinical tool based on the Framingham risk equation and optimised for an Australian populationthe Australian absolute CVD risk calculator. ${ }^{26}{ }^{27}$ This tool provides a 5-year risk, and the value doubled to estimate 10-year risk.

Patients will be excluded if they do not have an active mobile phone, are unable to read English and if they have documented CAD at the time of recruitment (defined as prior myocardial infarction, coronary artery bypass grafting, percutaneous coronary intervention, $50 \%$ or greater stenosis in at least one major epicardial vessel on invasive coronary angiography), or unable to provide informed consent.

\section{Randomisation and blinding}

Randomisation will occur via a computerised randomisation programme that will be accessed through a secure web interface by study staff with username and password. The randomisation programme is concealed from research staff involved in patient recruitment. The random allocation sequence will be in a $1: 1$ allocation ratio with block sizes of 4,6 and 8 . To maintain blinding of study personnel, patients will be informed of their allocation in a text message sent after leaving RACC. Prior to any follow-up appointments, patients will also receive a text message to ask them not to reveal their allocation status to study personnel or clinicians.

\section{Intervention}

Participants will be randomly allocated to either control (standard care group) or intervention (TextMe2 group). The TextMe2 intervention group will receive standard care (as described below) in addition to four text messages delivered each week over a 6 -month period. The content of the text messages will focus on lifestyle modification and medication adherence.

\section{Usual care group}

The control group will receive standard care including face-to-face lifestyle advice and pharmacotherapy as prescribed in the RACC and as determined by their usual doctors for the duration of the study. Participants in this group will receive two messages only-a welcome message after randomisation and an exit message at the conclusion of the study thanking them for their involvement and reminding them that they will be contacted for a follow-up assessment and to not reveal their group allocation. Participants in this group will not receive any educational texts.

Trained research personnel blinded to the group allocation will conduct assessments at baseline and 6 months with face-to-face interviews. 


\section{TextMe2 message bank development and delivery}

The text message intervention builds on our previous work in developing text message-based interventions from the TEXT ME study. ${ }^{21}{ }^{28}$ The key considerations used to design the text message structure and content have previously been published. ${ }^{29}{ }^{30}$ In brief, this involved a multiphase process including: (1) drafting and refinement of messages based on behaviour change techniques, national guidelines and expert review including academics and clinicians, (2) message refinement based on consumer feedback, and (3) software development and pilot testing. ${ }^{29}$

The TextMe2 message bank was developed for five topics: diet, physical activity, smoking, general cardiovascular health and medication adherence (box 1). Each patient will receive 100 educational messages over a 26-week period (four each week plus an entry and exit message). The majority of text messages providing information and support on lifestyle modification and medication adherence are from the TEXT $\mathrm{ME}^{28}$ and TEXT messages to improve MEDication adherence and Secondary prevention (TEXTMEDS) ${ }^{31}$ studies. Additionally, each participant will be prompted to rate every text message (except the entry and exit messages) they receive throughout the duration of the programme on a Likert scale between 1 (low) and 5 (best) (box 1). Participant's rating on the rating system will not affect the messages they receive. Future iterations may be used to further customise future programmes.

The TEXT ME and TEXTMEDS message banks were reviewed (164 and 192 messages, respectively) and the TextMe2 message bank refined for a primary prevention cohort (figure 2). Messages specific to a secondary prevention cohort (15 messages), messages containing reference to programmes no longer existing and duplicates were removed. For example, 'Hi usually it's safe to start mild exercise early after a heart event but if you are unsure ask your Dr or hospital cardiac rehab unit' and 'Don't forget to keep taking aspirin. It reduces the risk of further heart attacks after the first one by about $25 \%$, that's 1 in 4 chance' are specific to a secondary prevention cohort and thus removed. Existing messages were edited to reflect updated guidelines/trends or links to external resources. The Heart Foundation of Australia resources were reviewed, and 50 new messages developed. $^{32}{ }^{33}$ Given the increasing ubiquity of smartphones, messages were enhanced with links to external resources embedded within the texts with the final message bank including 6 weblinks to pictures, 7 video weblinks and 27 weblinks to additional reading material. All hyperlinks were changed to a shortened URL (uniform resource locator). Participant responses from TEXTMEDS ${ }^{31}$ were reviewed and suggestions were incorporated into the editing of existing messages (grammatical or spelling errors) and new messages included which reflect advice on alcohol consumption.

All new text messages underwent both expert (clinical academic, cardiologist and physiotherapist) and
Box 1 Examples of messages sent to the TextMe2 intervention group

General cardiovascular health

- Health professionals recommend 2 or less alcoholic drinks a day is ok, but less alcohol is recommended if you have high blood pressure, liver disease, diabetes, or if obese. Visit https://bit.ly/2CF1Rom to learn more. Please rate me 1 (low) to 5 (best).

- Hi [pref_name], has your doctor checked and discussed your cholesterol levels with you recently? These need regular review. Watch https://bit.ly/1AhJbWz to find out why. Please rate me 1 (low) to 5 (best).

> Did you know on average 22 Australians die from a heart attack each day? This is equivalent to 1 death from a heart attack every 66 minutes. Please rate me 1 (low) to 5 (best).

Diet

- [pref_name], salt is listed as sodium on food packaging. Visit https:// bit.ly/2txSM9v to check if you eat more than the recommended $4 \mathrm{~g}$ per day. Please rate me 1 (low) to 5 (best).

- The biggest proportion of your meal should be cooked vegetables or salad. Fill more than half of your plate with these foods [pref_name]. Visit https://bit.ly/2A9p9R0 for a guide. Please rate me 1 (low) to 5 (best).

- Make a few swaps when eating out. Swap a heavy to light meal option, eg, order tomato-based vs a cream-based pasta sauce or choose grilled over fried. Please rate me 1 (low) to 5 (best).

Physical activity

- [pref_name], have you got a pedometer or activity tracker? 10000 steps is the recommended daily step target for healthy adults. Visit https://bit.ly/2n69Hia to help you. Please rate me 1 (low) to 5 (best).

- Hi [pref_name], regular exercise can improve your blood pressure \& cholesterol. Visit https://bit.ly/2CKXrMU to learn more. Please rate me 1 (low) to 5 (best).

- Hi [pref_name], did you exercise today? Visit https://bit.ly/2F1ELtP to help get you motivated. Please rate me 1 (low) to 5 (best).

Smoking

- [pref_name], check out the website www.icanquit.com.au for tips \& to track your progress when quitting smoking. Did you like this message? Please reply with a rating from 1 to 5 stars.

- Hi [pref_name], smoking increases the risk of heart disease by up to 6 times. Watch https://bit.ly/2Nr3sFz to find out more. Please rate me 1 (low) to 5 (best).

- The Heart Foundation does not support using e-cigarettes. It's better to stop smoking altogether. Visit https://bit.ly/2yjSdVW to find out more. Please rate me 1 (low) to 5 (best).

\section{Medication adherence}

- Hi [pref_name], remember to take your medicines each day as recommended by your doctor or pharmacist. Visit https://bit.ly/2BT7KWT for tips. Please rate me 1 (low) to 5 (best).

- Hi [pref_name], are you still taking your medications every day? Keep it up! Visit https://bit.ly/2BYP66v for interesting facts. Please rate me 1 (low) to 5 (best).

- Hi [pref_name], did you know taking blood pressure and cholesterol medicines can halve your risk of a heart attack? Please rate me 1 (low) to 5 (best).

consumer review (adults of various sociodemographic backgrounds) - three reviewers in each group. Expert reviewers were asked to answer on a Likert scale of 1-5 whether: (1) they felt the content of the message aligned 


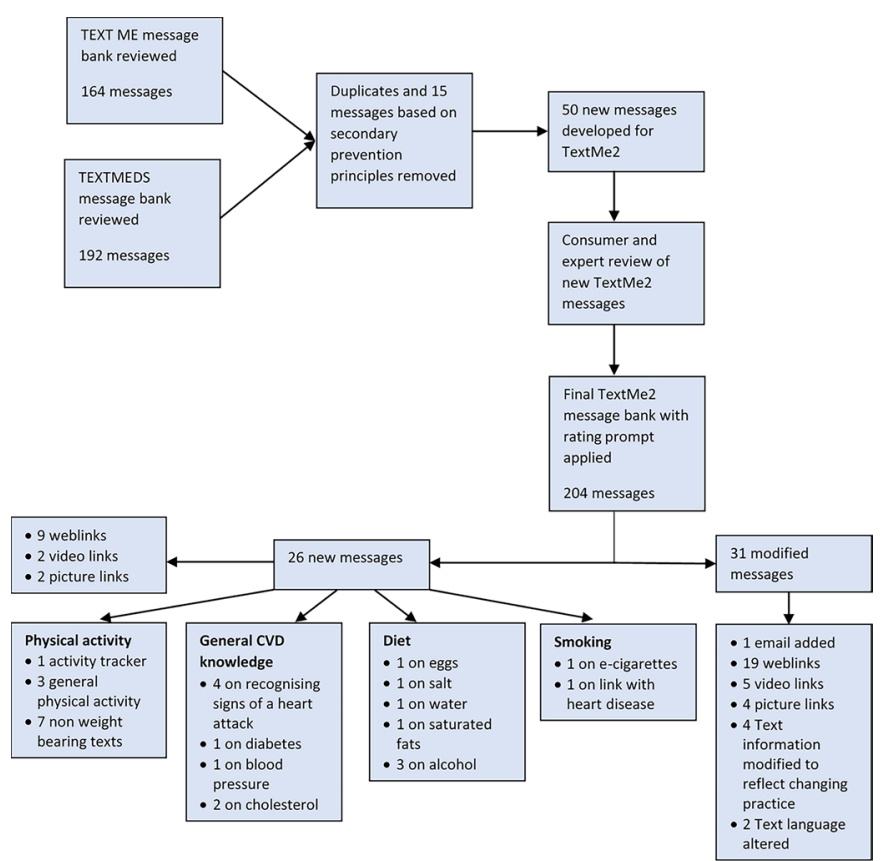

Figure 2 TextMe2 message bank development. CVD, cardiovascular disease; TEXT ME, Tobacco, Exercise and Diet Messages; TEXTMEDS, TEXT messages to improve MEDication adherence and Secondary prevention.

with current guidelines, and (2) the message is likely to be helpful to their patients. Any messages with a mean rating less than 4 were either edited or removed. Additionally, we asked expert reviewers to rate the likely level of health literacy required, and messages rated as very high health literacy were reworded. Consumers were asked to answer on a Likert scale of $1-5$ whether: (1) the message was easy to understand, and (2) the information provided was useful. Any messages rated less than 4 were either edited or removed.

The final TextMe2 message bank included 204 text messages in total (200 educational messages and four entry and exit messages). Each participant will receive 100 educational messages (of which 25 have links to educational resources) over a 26 -week period in addition to an entry and exit message. Messages will be sent four times per week from Monday to Friday between 09:00 and 17:00. The messages are drawn from four modules and across five topics (see table 1) each containing 25 messages: (1) general, (2) nutrition, (3) physical activity, (4) smoking alternating with (5) medication adherence. These will be delivered randomly within each module such that each week one message will be delivered from each module. The order of modules delivered each week will also be random. By the end of the programme all messages would have been delivered once only. The programme is semipersonalised such that vegetarian participants will receive diet messages for a vegetarian cohort; physical activity messages will be tailored depending on level of physical capacity (full activity, unable to weight-bear or unable to exercise); smokers will receive text messages regarding smoking cessation; participants taking cardiometabolic

\begin{tabular}{|c|c|c|}
\hline Module 1 & $\begin{array}{l}\text { General cardiovascular } \\
\text { knowledge }\end{array}$ & 9 weblinks \\
\hline Module $2 a$ & Standard diet & 4 weblinks \\
\hline Module 2b & Vegetarian diet & 4 weblinks \\
\hline Module $3 a$ & $\begin{array}{l}\text { Capable of full physical } \\
\text { activity }\end{array}$ & 7 weblinks \\
\hline Module 3b & $\begin{array}{l}\text { Non-weight-bearing } \\
\text { activity only }\end{array}$ & 7 weblinks \\
\hline Module 3c & Unable to exercise & 7 weblinks \\
\hline Module 4a & Current smoker & 3 weblinks \\
\hline Module $4 b$ & $\begin{array}{l}\text { Taking cardiometabolic } \\
\text { medications }\end{array}$ & 2 weblinks \\
\hline Module 4c & Non-smoker & 3 weblinks \\
\hline Module 4d & $\begin{array}{l}\text { Not taking } \\
\text { cardiometabolic } \\
\text { medications }\end{array}$ & 2 weblinks \\
\hline
\end{tabular}

medications will receive messages regarding medication adherence. Participants unable to exercise, non-smokers and those not taking cardiometabolic medications will receive generally cardiovascular texts instead. Messages will include the participants' preferred name and no message will be repeated. Study participants will not be encouraged to reply to text messages other than being prompted to rate every educational message they receive. However, replies will be monitored, and any questions asked from participants will be addressed by study staff.

Message delivery will be managed by computerised mHealth software (TextQStream, Python V.3.6 using Pycap V.1.02 library) as previously used. ${ }^{34}$ Research Electronic Data Capture (REDCap) ${ }^{35} 36$ will be used as a user interface to manage message delivery and customisation (smoking status, vegetarian or not vegetarian, physical activity ability and whether taking cardiometabolic medications). The REDCap user interface will keep a log of all messages sent to each study participant. The messaging engine will send messages to any Australian phone network at no cost to the participant and at a cost to the study. Data exports will be compliant with privacy legislation and centrally managed by Western Sydney Local Health District. There will be no access to data by any third party.

\section{Study outcomes and data collection}

Patients will be followed up at 6 months with a face-to-face interview with a researcher who will be blinded to treatment allocation. The primary outcome (table 2) is per cent change in the proportion of patients who have three or more uncontrolled modifiable risk factors (LDL-C $>2.0$ $\mathrm{mmol} / \mathrm{L}$, systolic blood pressure $>140 \mathrm{~mm} \mathrm{Hg}$, BMI $>24.9$ $\mathrm{kg} / \mathrm{m}^{2}$, physical activity (less than the equivalent of 150 min of moderate intensity each week), current smoker).

Secondary outcomes are changes in single biomedical risk factors, behavioural risk factors, quality of life, 


\begin{tabular}{|c|c|}
\hline Primary & $\begin{array}{l}\text { Per cent change in the proportion of patients who have three or more out of five uncontrolled modifiable risk } \\
\text { factors (LDL-C }>2.0 \mathrm{mmol} / \mathrm{L} \text {, systolic blood pressure }>140 \mathrm{~mm} \mathrm{Hg} \text {, BMI }>24.9 \text {, physical activity (less than the } \\
\text { equivalent of } 150 \mathrm{~min} \text { of moderate intensity each week), current smoker) }\end{array}$ \\
\hline Secondary & $\begin{array}{l}\text { Fasting LDL-C } \\
\text { Systolic blood pressure (average of two readings) } \\
\text { BMI and waist circumference } \\
\text { Change in HbA1c (for those with diabetes) } \\
\text { Global Physical Activity Questionnaire (GPAQ) and time spent in different domains } \\
\text { Self-reported smoking rate (cigarettes per day in last } 7 \text { days) and quit attempts (if relevant) } \\
\text { Servings of vegetables and fruits consumed each day (self-report of portions consumed in last } 7 \text { days) } \\
\text { Quality of life as assessed by the EQ-5D-5L Health Survey }{ }^{40} \\
\text { Depression score as assessed by Patient Health Questionnaire-9 (PHQ-9) }{ }^{41} \\
\text { Generalized Anxiety Disorder } 7 \text {-item (GAD-7) score }{ }^{42} \\
\text { Cardiovascular health literacy } \\
\text { Cardiometabolic medication adherence-self-reported }\end{array}$ \\
\hline
\end{tabular}

BMI, body mass index; HbA1c, glycosylated haemoglobin; LDL-C, low-density lipoprotein-cholesterol.

depression/anxiety scores, medication adherence, cardiovascular health literacy and hospital readmissions/ representations (table 2). Waist circumference will be assessed according to sex (high risk is $>102 \mathrm{~cm}$ in men and $>88 \mathrm{~cm}$ in women). Guideline recommendation of vegetable and fruit intake is $\geq 5$ serves and $\geq 2$ serves, respectively. Recommended levels of exercising defined as the equivalent of $150 \mathrm{~min}$ of moderate-intensity exercise each week. ${ }^{37}$ Glycosylated haemoglobin (HbAlc) will be recorded at the 6 months' follow-up and mean values calculated. The diagnosis of new diabetes will be made if two consecutive HbAlc levels measured at baseline are $\geq 6.5 \%$, two consecutive baseline fasting plasma glucose levels are $\geq 7.0 \mathrm{mmol} / \mathrm{L}$ or if both the baseline HbAlc and fasting plasma glucose are above their diagnostic thresholds as per current Australian recommendations. ${ }^{38}$ The Global Physical Activity Questionnaire comprises 16 questions, was developed by WHO and collects information across four domains (activity at work, travel to and from places, recreation and sedentary behaviour). ${ }^{39}$ Quality of life will be measured using the EQ-5D-5L developed by the EuroQol Group in $2009,{ }^{40}$ depression via the Patient Health Questionnaire- $9^{41}$ and anxiety via the Generalized Anxiety Disorder 7-item scale. ${ }^{42}$ Health literacy will be assessed at baseline using the BRIEF Health Literacy ${ }^{43}$ survey, and cardiovascular health literacy assessed at 6 months using a survey previously developed and peer reviewed (table 3). ${ }^{44}$ An endpoint adjudication process will be implemented and includes a review of medical records for major cardiovascular events, hospital admissions and deaths. Serious adverse events will be collected by the investigator throughout the duration of the study and reported to the project management committee and to ethics as per local guidelines. Multiple attempts will be made to contact participants to optimise follow-up including contacting their regular doctors.

Study data will be collected and managed using REDCap electronic data capture tools hosted at the University of Sydney. ${ }^{35}{ }^{36}$ REDCap is a secure, web-based software platform designed to support data capture for research studies, providing (1) an intuitive interface for validated data capture; (2) audit trails for tracking data manipulation and export procedures; (3) automated export procedures for seamless data downloads to common statistical packages; and (4) procedures for data integration and interoperability with external sources.

\section{Statistical considerations}

Analysis will be according to the intention-to-treat principle. Characteristics will be compared between the two groups using independent t-tests for continuous variables (LDL-C, systolic blood pressure, BMI, waist circumference, HbAlc, quality of life scores, health literacy) or $\chi^{2}$ tests for categorical variables (comparing proportions, smoking status, medication adherence, diet, physical activity, hospital readmissions and cardiac events). MannWhitney $\mathrm{U}$ tests will be used where data are not normally distributed. A significance level of $5 \%$ will be used. A full statistical analysis plan will be finalised prior to database lock.

\section{Sample size calculation}

During a 12-month enrolment period, based on our current referrals of moderate to high-risk patients without documented CAD (as defined by an absolute cardiovascular risk ${ }^{26} 27$ of greater than or equal to $10 \%$ ), and allowing for $10 \%$ loss to follow-up as per TEXT ME, ${ }^{28}$ our estimated possible sample size would be 246 . Using existing local control data ${ }^{25}$ we found that $62 \%$ of a primary prevention cohort attending an outpatient cardiology clinic had three or more risk factors. We estimated that with $90 \%$ power and two-sided $\alpha$ at 0.05 , a sample size of 246 (123 in each group) would be able to detect a $33 \%$ decrease in the proportion of people with three or more uncontrolled modifiable risk factors. 
Table 3 Data collection

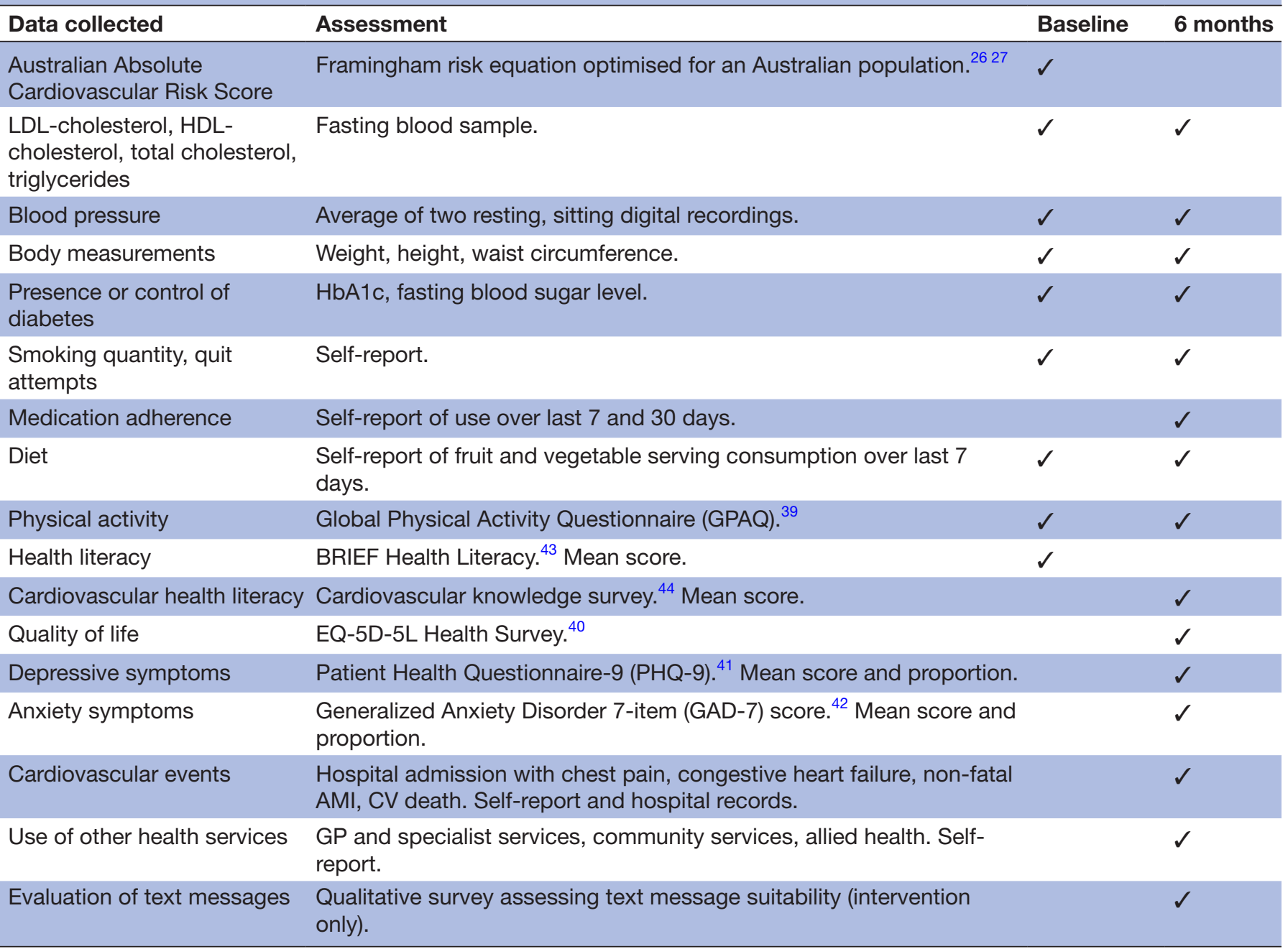

AMI, acute myocardial infarction; CV, cardiovascular ; GP, general practitioner; HbA1c, glycosylated haemoglobin; HDL, high-density lipoprotein; LDL, low-density lipoprotein.

The first patient was recruited on 3 June 2019 with estimated study completion by 28 December 2020 .

\section{Process evaluation}

For process measures, an electronic log will be automatically kept assessing the time messages are delivered and the status of the delivery (ie, delivered or not delivered). Additionally, a log will be kept of responses received from participants and when participants contact the study team including the reason for contact and the method used. A 'screening log' of reasons for non-participation will be kept for those ineligible or who decline to participate.

To examine the acceptability and feasibility of TextMe2, all participants in the intervention group at the 6-month follow-up period will be asked to complete a structured questionnaire of a series of Likert responses about their experiences of being sent health text messages. Questions will address the understanding and tolerability of the text messages, asking which the participants liked or disliked, perceived utility and intrusiveness. Engagement with
TextMe2 will be assessed by asking participants if they felt the text messages motivated them to change their lifestyle, helped remind them to take their medications and whether they rated the text messages as requested. In addition, we will ask the proportion of messages that were read and whether messages were shown to family/ friends and/or forwarded. Finally, the proportion of patients responding to messages with a rating will be used as a marker of overall engagement.

\section{Patient and public involvement}

The majority of text messages have previously been tested and assessed for acceptability in the TEXT $\mathrm{ME}^{21}$ and TEXTMEDS ${ }^{31}$ trials. Patient feedbacks from these studies were used to modify message content for TextMe2. Additionally, three patients and three healthcare professionals (allied health, clinical academic and cardiologist) were surveyed prior to study initiation to critically review new messages included in TextMe2 as detailed above. 


\section{ETHICS AND DISSEMINATION}

The study will be administered at Westmead Hospital with the design and conduct overseen by a project management committee (authors) from The University of Sydney's Westmead Applied Research Centre. This committee has expertise in large-scale clinical trials, qualitative research and clinical CVD management. This study will adhere to the National Health and Medical Research Council ethical guidelines for human research. Ethics approval for this study has been obtained from the Western Sydney Local Health Network Human Research Ethics Committee (HREC/17/WMEAD/186). Written informed consent will be obtained from all enrolled participants (refer to online supplementary material 1).

Results of this study will be presented at national and international meetings and published in a scientific journal. Participants will not be individually notified regarding the results of the study.

\section{DISCUSSION}

TextMe2 will generate reliable data about the effectiveness of an entirely text message-based intervention as a potential primary prevention solution to control multiple cardiovascular risk factors among those at moderate to high cardiovascular risk. It will also provide information on implementation of such a programme in a primary prevention patient population. An mHealth solution such as this can provide scalable but semicustomised primary prevention support that could extend the reach and effectiveness of primary prevention programmes, but the challenge is maintaining user engagement. By employing a rating system with each message delivered, TextMe2 will also provide new evidence as to the characteristics of messages that are preferred by different participants and thus help further individualise future mHealth programmes potentially improving engagement.

We have previously demonstrated that text messagebased prevention programmes are effective and costeffective in CVD secondary prevention. ${ }^{12}$ Additionally, text message-based digital health interventions are easily automated and have the potential to bridge healthcare access gaps across different settings and evidence also supports their use in low-income countries and geographically isolated communities. ${ }^{13-15}$ Thus, if TextMe2 is found to be effective in a primary prevention setting, there is potential for scalability to improve CVD prevention at a population level. For instance, the Be He@lthy, Be Mobile initiative jointly founded by the WHO and the International Telecommunication Union helps governments across low, middle and high-income countries to introduce evidencebased mHealth services within government frameworks to manage non-communicable diseases and their risk factors including tobacco smoking and diabetes. ${ }^{45}$ Additionally, the SmokefreeTXT ${ }^{46}$ government-supported programme has been integrated nationally within the USA following strong evidence supporting the use of text messages for smoking cessation. ${ }^{16}$
Better preventive care in individuals with moderate-high absolute cardiovascular risk will decrease incident cases of CVD and thus healthcare service utilisation and associated costs. Yet optimising engagement with current preventive strategies in this population is challenging. The delivery of text message-based programmes in combination with cardiovascular risk assessment at the clinical interface can motivate and empower patients to self-care and make healthy behaviour change.

This study has some limitations. Smoking cessation, diet, physical activity and medication adherence are assessed using self-report. However, self-report is the simplest, most inexpensive and most relevant way to assess lifestyle risk factors in the clinic setting. We acknowledge that a 6-month period is a short-term follow-up and the study is single site and has a small sample size; however, TextMe2 will provide preliminary data essential to design larger multicentre trials with longer follow-up.

\section{Twitter Harry Klimis @HarryKlimis}

Acknowledgements This study is supported by the Western Sydney Local Health District and the University of Sydney. We thank research assistant Daniel McIntyre for assisting with REDCap database and evaluation of video links included in the text messages, and Professor Julie Redfern for evaluating the text message bank. We also thank the patients who took their time evaluating the text messages, and the staff in the RACC for supporting patient recruitment.

Contributors HK, AT and CKC conceived the original study concept. All authors contributed to the design of the study, protocol development, its implementation and drafting of the manuscript.

Funding This study is funded by the National Heart Foundation Future Leader Fellowship programme. HK is supported by the Royal Australian College of Physicians (RACP) Fellows Research Entry Scholarship. CC is supported by the National Health and Medical Research Council (NHMRC) Career Development Award (APP1105447) cofunded by the National Heart Foundation.

\section{Competing interests None declared.}

Patient and public involvement Patients and/or the public were involved in the design, or conduct, or reporting, or dissemination plans of this research. Refer to the Methods section for further details.

\section{Patient consent for publication Not required.}

Provenance and peer review Not commissioned; externally peer reviewed.

Open access This is an open access article distributed in accordance with the Creative Commons Attribution Non Commercial (CC BY-NC 4.0) license, which permits others to distribute, remix, adapt, build upon this work non-commercially, and license their derivative works on different terms, provided the original work is properly cited, appropriate credit is given, any changes made indicated, and the use is non-commercial. See: http://creativecommons.org/licenses/by-nc/4.0/.

ORCID iD

Harry Klimis http://orcid.org/0000-0002-3635-421X

\section{REFERENCES}

1 World Health Organisation. Media centre: cardiovascular diseases (CVDs), 2015. Available: http://www.who.int/mediacentre/factsheets/ fs317/en/ [Accessed 18 Feb 2016].

2 Australian Institute of Health and Welfare. Monitoring acute coronary syndrome using national hospital data: an information paper on trends and issues. Canberra: AlHW, 2011.

3 Yusuf S, Hawken S, Ounpuu S, et al. Effect of potentially modifiable risk factors associated with myocardial infarction in 52 countries (the INTERHEART study): case-control study. Lancet 2004;364:937-52.

4 Piepoli MF, Hoes AW, Agewall S, et al. 2016 European Guidelines on cardiovascular disease prevention in clinical practice: The Sixth Joint Task Force of the European Society of Cardiology and Other Societies on Cardiovascular Disease Prevention in Clinical Practice 
(constituted by representatives of 10 societies and by invited experts) Developed with the special contribution of the European Association for Cardiovascular Prevention \& Rehabilitation (EACPR). Eur Heart $J$ 2016;37:2315-81.

5 Arnett DK, Blumenthal RS, Albert MA, et al. 2019 ACC/AHA guideline on the primary prevention of cardiovascular disease: a report of the American College of Cardiology/American heart association Task force on clinical practice guidelines. Circulation 2019;140:e596-646.

6 Karmali KN, Persell SD, Perel P, et al. Risk scoring for the primary prevention of cardiovascular disease. Cochrane Database Syst Rev 2017;3:CD006887.

7 Gupta R, Wood DA. Primary prevention of ischaemic heart disease: populations, individuals, and health professionals. Lancet 2019;394:685-96.

8 Bastawrous A, Hemming B, Livingstone I. mHealth possibilities in a changing world. distribution of global cell phone Subscriptions. $J$ Mob Technol Med 2013;2:22-5.

9 Burke LE, Ma J, Azar KMJ, et al. Current science on consumer use of mobile health for cardiovascular disease prevention: a scientific statement from the American heart association. Circulation 2015;132:1157-213.

10 World Health Organisation. mHealth: new horizons for health through mobile technologies: second global survey on eHealth Geneva. Switzerland: World Health Organization, 2011. http://www.who.int/ goe/publications/goe_mhealth_web.pdf

11 Klimis H, Thakkar J, Chow CK. Breaking barriers: mobile health interventions for cardiovascular disease. Can J Cardiol 2018;34:905-13.

12 Burn E, Nghiem S, Jan S, et al. Cost-Effectiveness of a text message programme for the prevention of recurrent cardiovascular events. Heart 2017:103:893.1-4.

13 Thakkar J, Karthikeyan G, Purohit G, et al. Development of macaronic Hindi-English 'Hinglish' text message content for a coronary heart disease secondary prevention programme. Heart Asia 2016;8:32-8.

14 Zheng X, Spatz ES, Bai X, et al. Effect of text messaging on risk factor management in patients with coronary heart disease: the CHAT randomized clinical trial. Circ Cardiovasc Qual Outcomes 2019;12:e005616.

15 Kumar D, Raina S, Sharma SB, et al. Effectiveness of randomized control trial of mobile phone messages on control of fasting blood glucose in patients with type-2 diabetes mellitus in a northern state of India. Indian J Public Health 2018;62:224-6.

16 Whittaker R, McRobbie H, Bullen C, et al. Mobile phone-based interventions for smoking cessation. Cochrane Database Syst Rev 2016;4:CD006611.

17 Siopis G, Chey T, Allman-Farinelli M. A systematic review and meta-analysis of interventions for weight management using text messaging. J Hum Nutr Diet 2015;28 Suppl 2:1-15.

18 Arambepola C, Ricci-Cabello I, Manikavasagam P, et al. The impact of automated brief messages promoting lifestyle changes delivered via mobile devices to people with type 2 diabetes: a systematic literature review and meta-analysis of controlled trials. J Med Internet Res 2016;18:e86.

19 Thakkar J, Kurup R, Laba T-L, et al. Mobile telephone text messaging for medication adherence in chronic disease: a meta-analysis. JAMA Intern Med 2016;176:340-9.

20 Klimis $\mathrm{H}$, Khan ME, Kok C, et al. The role of text messaging in cardiovascular risk factor optimization. Curr Cardiol Rep 2017;19:4.

21 Chow CK, Redfern J, Hillis GS, et al. Effect of Lifestyle-Focused text messaging on risk factor modification in patients with coronary heart disease: a randomized clinical trial. JAMA 2015;314:1255-63.

22 Anand SS, Samaan Z, Middleton C, et al. A digital health intervention to lower cardiovascular risk: a randomized clinical trial. JAMA Cardiol 2016;1:601-6.

23 Liu Z, Chen S, Zhang G, et al. Mobile Phone-Based lifestyle intervention for reducing overall cardiovascular disease risk in Guangzhou, China: a pilot study. Int J Environ Res Public Health 2015;12:15993-6004.

24 Klimis H, Thiagalingam A, Altman M, et al. Rapid-access cardiology services: can these reduce the burden of acute chest pain on
Australian and New Zealand health services? Intern Med J 2017:47:986-91.

25 Klimis $\mathrm{H}$, Khan ME, Thiagalingam A, et al. Rapid access cardiology (Rac) services within a large tertiary referral Centre-First year in review. Heart Lung Circ 2018;27:1381-7.

26 National Vascular Disease Prevention Alliance. Australian absolute cardiovascular disease risk calculator, 2012. Available: cvdcheck.org. au

27 Anderson KM, Odell PM, Wilson PW, et al. Cardiovascular disease risk profiles. Am Heart J 1991;121:293-8.

28 Chow CK, Redfern J, Thiagalingam A, et al. Design and rationale of the tobacco, exercise and diet messages (text me) trial of a text message-based intervention for ongoing prevention of cardiovascular disease in people with coronary disease: a randomised controlled trial protocol. BMJ Open 2012;2:e000606.

29 Redfern J, Thiagalingam A, Jan S, et al. Development of a set of mobile phone text messages designed for prevention of recurrent cardiovascular events. Eur J Prev Cardiol 2014;21:492-9.

30 Thakkar J, Barry T, Thiagalingam A, et al. Design considerations in development of a mobile health intervention program: the text me and TEXTMEDS experience. JMIR Mhealth Uhealth 2016;4:e127.

31 Chow CK, Thiagalingam A, Santo K, et al. TEXT messages to improve MEDication adherence and Secondary prevention (TEXTMEDS) after acute coronary syndrome: a randomised clinical trial protocol. BMJ Open 2018;8:e019463.

32 National Heart Foundation of Australia. Available: https://www. heartfoundation.org.au/for-professionals

33 National Heart Foundation of Australia. My heart, my life, 2017.

34 Stevenson J, Campbell KL, Brown M, et al. Targeted, structured text messaging to improve dietary and lifestyle behaviours for people on maintenance haemodialysis (KIDNEYTEXT): study protocol for a randomised controlled trial. BMJ Open 2019;9:e023545.

35 Harris PA, Taylor R, Minor BL, et al. The REDCap Consortium: building an international community of software platform partners. $J$ Biomed Inform 2019;95:103208.

36 Harris PA, Taylor R, Thielke R, et al. Research electronic data capture (REDCap)--a metadata-driven methodology and workflow process for providing translational research informatics support. J Biomed Inform 2009;42:377-81.

37 Arnett DK, Blumenthal RS, Albert MA, et al. 2019 ACC/AHA guideline on the primary prevention of cardiovascular disease: a report of the American College of Cardiology/American heart association Task force on clinical practice guidelines. Circulation 2019;140.

38 Zheng Q, Chen C, ZN L, et al. A novel Multi-Resolution SVM (MRSVM) algorithm to detect ECG signal anomaly in WE-CARE project. 2013 Issnip Biosignals and Biorobotics conference. New York: leee, 2013: 28-33.

39 WHO. Global physical Activty questionnaire (GPAQ), 2017. Available: http://www.who.int/chp/steps/GPAQ/en/2017 [Accessed 30 Mar 2017].

40 Herdman M, Gudex C, Lloyd A, et al. Development and preliminary testing of the new five-level version of EQ-5D (EQ-5D-5L). Qual Life Res 2011;20:1727-36.

41 Kroenke K, Spitzer RL, Williams JB. The PHQ-9: validity of a brief depression severity measure. J Gen Intern Med 2001;16:606-13.

42 Spitzer RL, Kroenke K, Williams JBW, et al. A brief measure for assessing generalized anxiety disorder: the GAD-7. Arch Intern Med 2006;166:1092-7.

43 Haun J, Luther S, Dodd V, et al. Measurement variation across health literacy assessments: implications for assessment selection in research and practice. $J$ Health Commun 2012;17 Suppl 3:141-59.

44 Bergman HE, Reeve BB, Moser RP, et al. Development of a comprehensive heart disease knowledge questionnaire. Am J Health Educ 2011;42:74-87.

45 World Health Organisation and International Telecommunciation Union. Be He@lthy Be Mobile, 2019. Available: https://www.who.int/ ncds/prevention/be-healthy-be-mobile/en/2019 [Accessed 6th Dec 2019].

46 National Institutes of Health. Smokefree.gov, 2019. Available: https:// smokefree.gov/ [Accessed 6 Dec 2019]. 